\title{
INFLUENCE OF THE ELASTO-PLASTIC BEHAVIOR OF TIE-RODS IN THE RESPONSE OF ROCKING MASONRY WALLS THROUGH SEISMIC DEMAND HAZARD CURVES
}

\author{
F. Solarino ${ }^{1}$, L. Giresini ${ }^{2 *}$, and P. Croce ${ }^{3}$ \\ ${ }^{1}$ Department of Energy, Systems, Territory and Constructions Engineering \\ University of Pisa \\ Largo L. Lazzarino, 1, 56126 Pisa \\ e-mail: f.solarino1@ studenti.unipi.it \\ $2 *$ corresponding author. Department of Civil and Industrial Engineering \\ University of Pisa \\ Largo L. Lazzarino, 1, 56126 Pisa \\ email: linda.giresini@unipi.it \\ ${ }^{3}$ Department of Civil and Industrial Engineering \\ University of Pisa \\ Largo L. Lazzarino, 1, 56126 Pisa \\ email: p.croce@ing.unipi.it
}

\begin{abstract}
Rocking analysis is a powerful tool to assess the seismic vulnerability assessment of masonry walls subjected to out-of-plane modes, especially when in view of checking the efficiency of traditional retrofitting solutions, such as steel tie-rods restraining rocking blocks. The study focuses on a probabilistic approach for the seismic assessment of the out-of-plane behavior of masonry walls, mainly aiming to reliably predict fragility and seismic demand hazard curves in case steel tie rods are used as anti-seismic device. To identify the most appropriate steel tie-rod device, more than thousand multistripe analyses have been performed considering the Italian site with highest seismic hazard (Carlentini, Sicily), duly modifying ductility and strength of the tie rods themselves. The resulting fragility curves and seismic demand hazard curves are critically discussed, so allowing the definition of the most efficient and proficient intensity measures referring to five relevant limit states. As expected, remarkable changes in the response are recorded by passing from a brittle to a ductile tie-rod, but when the ultimate strain is bigger than $2 \%$, an increased tie-rod ductility does not sensitively improve the response even for highintensity earthquakes. The probabilistic approaches show that even low-ductility tie-rods can sensibly reduce the probability of exceedance of limited and moderate rocking limit states, up to an order of magnitude. As for the influence of the tie-rod strength, even low-medium values produce a remarkable reduction of annual exceedance rate. For instance, a severe rocking limit state occurs for the unrestrained monumental wall every 450 years and every 2000 years for the wall restrained by a tie rod of strength just fitting the minimum required design value.
\end{abstract}


Keywords: rocking, brittle tie-rod, ductile tie-rod, historic masonry, annual exceedance rate, restrained walls, out-of-plane. 


\section{INTRODUCTION}

Masonry structures can be seen as an assembly of macro-elements. Independently on the masonry type [1] and mechanical properties [2], these macro-elements that can be considered as a rigid system, provided that the masonry is monolithic. The rocking analysis of rigid blocks is a non-linear dynamic analysis capable of simulating the behavior of masonry walls rocking out-of-plane. The out-of-plane behavior is undesired for masonry buildings but unfortunately very common for the lack of proper connections between walls and between walls and horizontal diaphragms. Owing the fact that many types of such connections were developed over the centuries, ad hoc detailed studies are necessary to understand their effectiveness [3].

Nowadays, a wide literature is available on rocking analyses of free-standing walls as well as vertically/horizontally restrained walls, including analytical formulations [4]-[9], experimental outcomes [10]-[13] and numerical approaches [5], [14]-[18]. All these contributions are aimed at reliably representing the dynamic behavior of a masonry wall rocking out-of-plane during an earthquake.

Horizontal restraints are often provided by steel tie-rods, traditionally used in historic masonry buildings [19]-[21]. The response of free-standing blocks is strongly influenced by the size and slenderness ratio of the wall, besides the frequency content and numerous intensity measures associated to the seismic input. In addition to these parameters, the dynamic response of blocks restrained by horizontal tie-rods depends on the position and on the mechanical properties of the tie-rods themselves, as discussed in [22]. In [22] it was demonstrated on one hand that, as expected, tie-rods reduce the maxima amplitude rotations, but on the other hand that tied walls behavior is not significantly dependent on the aspect ratio of two walls of comparable size. The latter remark is still valid for walls restrained by tie-rods at different heights; more generally, it can be observed that the influence of tie-rod length as well as of normalized prestress is very limited. As for the influence of the mechanical properties, the role of the axial stiffness appears to be negligible. The yield strength was the most relevant parameter that influences the response, besides the brittle or ductile behavior of the tie-rod.

This contribution takes inspiration from the cited work but it considers different geometries and assumptions for the tie-rods. Moreover, this paper adopts a full stochastic approach for the seismic vulnerability assessment [23]-[26], whereas in [22] a semi-probabilistic approach is considered. After the presentation of the analytical formulation (Section 2) and the assumptions (Section 3) taken into account in the analysis, the work discusses the results in terms of fragility curves (Section 4) and seismic hazard curves (Section 5), which is a new frontier in the probabilistic assessment of seismic vulnerability [27].

\section{ANALYTICAL FORMULATION}

\subsection{Mechanical model and equation of motion}

The out-of-plane behavior of existing masonry structures can be investigated through nonlinear dynamic analyses also called rocking analyses. This section describes the model considered for analyzing the one-sided motion of masonry walls restrained by steel tie-rods, displayed in Figure 1. In it, the wall is assumed as single degree of freedom rigid block of mass $m$ and of perpendicular shape. Its size and slenderness are respectively given by $R$ and $\alpha=\arctan \left(\frac{h}{b}\right)$. The equation of motion, found in previous works of the Authors [14], [15], [17], is the following:

$$
I_{0} \ddot{\vartheta}+\operatorname{sgn}(\vartheta) m g R \sin A_{\vartheta}+T_{t}+T_{L}=m \ddot{u}_{g} R \cos A_{\vartheta}
$$


The right hand side contains the seismic input term as acceleration time history $\ddot{u}_{g}(t)$ of the earthquake. The terms on the left hand side are related to the inertia forces, the self-weight stabilizing the response up to a rotation threshold equal to $\alpha$, the tie-rod of stiffness $K\left(T_{t}\right)$ and the transverse walls simulated by a spring bed of unitary stiffness $K_{c}^{\prime}\left(T_{L}\right)$. As for the symbols, $I_{0}$ is the polar inertia moment with respect to the pivot point $\mathrm{O}$ or $\mathrm{O}^{\prime}$ equal to $\frac{4}{3} m R^{2}$, whereas $A_{\vartheta}=\alpha-\operatorname{sgn}(\vartheta) \vartheta$.
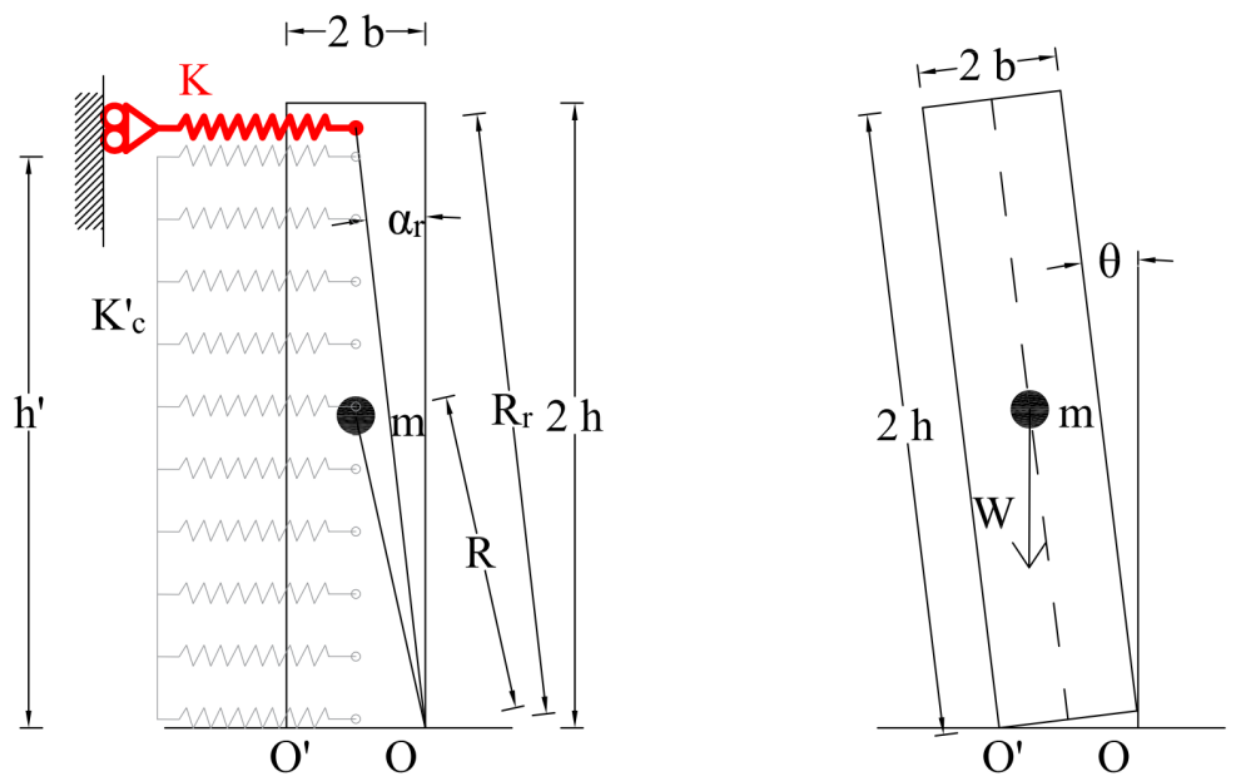

Figure 1: schematics of the model considered in the rocking analyses.

The term $T_{t}$ assumes the form:

$$
T_{t}=\operatorname{sgn}(\vartheta) K \beta^{2} R^{2} \cos A_{r, \vartheta}\left[\sin \alpha_{r}-\sin A_{r, \vartheta}\right]
$$

in which $A_{r, \vartheta}=\alpha_{r}-\operatorname{sgn}(\vartheta) \vartheta$ where $\alpha_{r}$ is a position angle dependent on the position coefficient $\beta=R_{r} / R$ (Figure 1). The tie-rod can behave either as elastic or as plasticized. If the tie-rod is in the elastic field, the corresponding term can be simply calculated from the external work made by it, obtaining:

$$
T_{t, e}=\left[\operatorname{sgn}(\vartheta) K R_{r}\left(\sin \alpha_{r}-\sin A_{r, \vartheta}\right)+F_{0}\right] R_{r} \cos A_{r, \vartheta}
$$

$T_{t, e}$ is considered in the equation of motion if the tie-rod displacement $d_{t}$ is in the range of $0 \leq d_{t} \leq d_{y}$ (the sub-index $y$ stands for yielding) or $d_{r} \leq d_{t} \leq d_{\max }$ (the sub-index max stands for ultimate displacement). The tie-rod can be subjected to a prestress $F_{0}$. Once yielding is attained, $d_{t}>d_{\max }$ or equivalently $d_{t}>d_{\max }$, the spring exerts the constant yielding force $F_{y}$. The term related to the plastic tie-rod contribution is then:

$$
T_{t, p}=\operatorname{sgn}(\vartheta) F_{y} R_{r} \cos A_{r, \vartheta}
$$

As usual, a compressed tie-rod is considered inactive. In case of unloading, the constitutive law follows the elastic branch. Anyway, the constitutive model is fully described in [28]. The tie-rod influence can be controlled through the following parameters: stiffness, yielding, ductility and strength associated to it.

$T_{L}$ is different from zero only for counterclockwise rotations (spring bed in compression, Figure 1). In that case it is expressed by[29]: 


$$
T_{L}=\operatorname{sgn}(\vartheta) K_{c}^{\prime} h^{\prime}\left(A+\frac{B h^{\prime}}{2}+\frac{C h^{\prime 2}}{3}\right)
$$

where:

$$
\begin{gathered}
A=\operatorname{sgn}(\vartheta) 4 b^{2} \sin \vartheta \cos \vartheta(1-\cos \vartheta) ; \\
B=2 b\left(\sin ^{2} \vartheta \cos \vartheta-\cos ^{3} \vartheta+\cos ^{2} \vartheta\right) ; \\
C=\operatorname{sgn}(\vartheta) \sin \vartheta \cos ^{2} \vartheta .
\end{gathered}
$$

$h^{\prime}$ is the extension of the spring bed, $2 b \mathrm{t}$ is the wall thickness and $K_{c}^{\prime}$ is the stiffness of the spring bed. $K_{c}^{\prime}$ can be calculated as function of the horizontal elastic modulus of masonry $E_{x}$, actual transverse wall length $L_{L, e f f}$ and transverse wall thickness $t_{t}$ according to the multistruts model presented in [29].

The solution of the equation of motion (Eq. (1)) gives the rotation time history $\vartheta(t)$ of the block and all the other parameters (acceleration, velocity, displacement) related to the center of mass and to the other significant points of the wall. The solution of the differential equation is performed by means of the ODE45 solver available in MATLAB [30], which uses a $4^{\text {th }}-5^{\text {th }}$ order Runge-Kutta integration technique.

\subsection{Probabilistic model for the determination of fragility curves and seismic hazard curves}

The probabilistic model employed for calculating the fragility curves is extensively described in [31] and that for obtaining the seismic hazard curves is illustrated in [23]. To be concise, only a general description of them is given in the following. The fragility curves are plotted by calculating the conditional probability of exceedance of specific limit states defined by the engineering demand parameter EDP $\vartheta / \alpha$. The EDPs, taken as maximum normalized rotation of each response time-history, clearly depend on the seismic input, in turn defined by specific intensity measures IMs. The conditional probability $P(E D P>C \mid I M)$ that the EDP overcomes a capacity threshold $C$ conditioned to an IM is expressed by:

$$
P_{e x}(E D P>C \mid I M)=\Phi\left(\frac{\ln D-\ln C}{\beta_{D \mid I M}}\right) .
$$

The capacity thresholds $C$ considered for the corresponding limit states are:

1. LS0, rocking initiation, $E D P>0.0$;

2. LS1, limited rocking, $E D P=0.1$;

3. LS2, moderate rocking, $E D P=0.4$;

4. LS3, severe rocking, $E D P=1.0$.

5. LS4, near-collapse rocking, $E D P=1.5$.

$\Phi$ is the standard cumulative distribution function $\Phi, \beta_{D \mid I M}$ is the logarithmic standard deviation, or dispersion, of the demand conditioned on the $I M$ level. $D$ is the median structural demand which for univariate fragility curves is equal to:

$$
D=a I M^{b} \text {. }
$$


$D$ coincides with the EDP coming from the rocking analyses, while $a$ and $b$ are the two correlation coefficients which derive from the post-processing of the results expressing $D$ in $\operatorname{loga}$ rithmic terms.

Preliminarily, the results of a set of rocking analyses with variable seismic inputs should be obtained. Each output of the rocking analysis, for a fixed geometry and fixed boundary conditions (mechanical properties of the tie-rods) is a single realization. These realizations are then elaborated to obtain the correlation coefficients. The larger is the correlation coefficient $b$, the more practical is the correlation with the intensity measure. The lower the dispersion coefficient $\beta_{D \mid I M}$, the more efficient is the correlation. A third correlation coefficient is $\zeta=\beta_{D \mid I M} / b$ to assess proficiency. The realizations are then used for calculating the fragility as conditional probability of failure [32], where the demand $D$ is represented by the EDPs calculated from the rocking analyses and the capacity $C$ is defined by the five limit states also expressed in [31], [33]. Different fragility curves can be plotted, depending on the fact that the case is categorical (rocking-non rocking) or a specific limit state is exceeded. In this paper the so-called "final probability" $P_{f}$ is plotted combining the probability of exceedance $P_{e x}$ to the probability of overturning $P_{R O}$. The complete procedure is described in [34].

\section{ASSUMPTIONS OF THE ANALYSIS}

This section illustrates the assumptions of the analysis in terms of choice of geometries, seismic inputs and mechanical properties of tie-rods. Indeed, all these parameters have an impact on the dynamic response.

\subsection{Wall geometries}

Two wall geometries are selected to give a first glimpse of their influence on the response. A medium-high slenderness ratio of $\alpha=0.1$ is chosen, corresponding to a ratio of wall height/thickness (conventional slenderness) equal to 10. Upper limit values of conventional slenderness are fixed to 12 for unreinforced brick masonry (10 for stone masonry). These limit values are valid for earthquake resistant walls [35]. For what concerns the wall size, two thickness values are considered: $30 \mathrm{~cm}$ and $60 \mathrm{~cm}$. Consequently, being $\alpha=0.1$, the two respective heights are $300 \mathrm{~cm}$ and $600 \mathrm{~cm}$. The walls have unit width and specific weight of $19 \mathrm{kN} / \mathrm{m}^{3}$. For the sake of clarity, one can define the first wall as ordinary wall, and the second one as monumental wall, since its greater size is similar to that of the main façades of historic or monumental buildings.

\subsection{Mechanical properties of the tie-rods and transverse walls}

This paper is mainly aimed at assessing the influence of the tie-rods in the dynamic response. The variability of mechanical properties is taken into account in terms of:

- Yield stress $f_{y}$ (which assumes the values 142-235-294 MPa);

- Area $A$ (calculated according to the procedures of kinematic linear analyses [35] assuming $\mathrm{PGA}=0.278 \mathrm{~g})$;

- $\quad$ Yielding force $F_{y}=f_{y} \cdot A$;

- Ultimate strain $\varepsilon_{u}$ (which assumes the values 0-2-10\%).

The yielding strength is that typical of existing tie-rods in unreinforced masonry structures, also historical [36]. The last parameter is related to the ductility of the tie-rod. This investigation has the purpose of analyzing its effect on the response depending on the level of ductility of the 
tie-rod material. It is physically expectable that a brittle tie-rod behaves differently from a ductile tie-rod, but the quantitative difference can be assessed only through a comparison of nonlinear dynamic analyses. Moreover, deterministic analyses are not fully reliable due to the fact that the response is input-dependent.

The response is reported in terms of a dimensionless parameter called restraining efficiency coefficient $\rho_{M, e}$. This parameter reads as ratio of a resisting moment $M_{r}$ to a seismic demand moment $M_{d}$. In particular, the resisting moment can be expressed as:

$$
M_{r}=F_{y} \cdot h_{r}
$$

where $h_{r}$ is the lever arm of the tie-rod, that is the distance of the tie-rod from the rocking hinge on the wall foundation. The seismic demand moment is calculated assuming an acceleration variable with the block height based on its first modal shape, whose maximum acceleration $a_{d}$ is assumed to be equal to the gravity acceleration:

$$
a_{d}=\ddot{\theta}(z=2 h) \cdot z=\frac{g}{2 h} z
$$

Considering the distance $z$ of the mass per unit of length $\mu$ from the rocking base hinge, the corresponding force is:

$$
d F_{d}=\mu \cdot a_{d} d z
$$

The seismic demand moment reads, considering Eq. (11) and Eq. (12):

$$
M_{d}=\int_{0}^{2 h} d F_{d} \cdot z=\int_{0}^{2 h} \mu \frac{g}{2 h} \cdot z^{2} d z .
$$

In the present case, being $\mu$ constant, it results:

$$
M_{d}=\frac{4}{3} W h
$$

where $W$ is the wall weight. Finally, when the tie is installed at the top of the wall, namely $h_{r}=$ $2 h$, the restraining efficiency coefficient $\rho_{M, e}$ assumes the following expression:

$$
\rho_{M, e}=\frac{M_{r}}{M_{d}}=\frac{3}{2} \frac{F_{y}}{W}
$$

In the present case, since the diameter of the tie-rod is calculated from the design criterion of the kinematic linear analysis [35], $\rho_{M, e}$ is constant for both cases (Table 1). By contrast, the consequent tie-rod diameter $\varphi$ varies as reported in Table 2, because the yielding strength changes.

\begin{tabular}{lrrr} 
& $W[\mathrm{kN}]$ & $F_{y}[\mathrm{kN}]$ & $\rho_{M, e}[-]$ \\
\hline ordinary wall & 17.1 & 5.7 & 0.5 \\
\hline monumental wall & 68.4 & 22.9 & 0.5
\end{tabular}

Table 1: Weight of the analyzed walls, yielding force and restraining efficiency coefficient of the tied configuration.

\begin{tabular}{lrrr}
$\boldsymbol{f}_{\boldsymbol{y}}[\mathbf{M P a}]$ & $\mathbf{1 4 2}$ & $\mathbf{2 3 5}$ & $\mathbf{2 9 4}$ \\
\hline ordinary wall & 7.2 & 5.6 & 5 \\
\hline monumental wall & 14 & 11 & 10
\end{tabular}

Table 2: Variation of tie-rod diameter (in $\mathrm{mm}$ ) with the yielding strength. 
As for the transverse walls, the following parameters are considered: extension of the spring bed $h^{\prime}=2 h$, horizontal elastic modulus of masonry $E_{x}=690 \mathrm{MPa}$, actual transverse wall length $L_{L, e f f}=1.2 \mathrm{~m}$ and transverse wall thickness $t_{t}=2 \mathrm{~b}$. By substituting the values for the two wall cases, one has $K_{c}^{\prime}=172.5 \mathrm{MN} / \mathrm{m}^{2}$ and $K_{c}^{\prime}=345.0 \mathrm{MN} / \mathrm{m}^{2}$ respectively for the ordinary wall and for the monumental wall.

\subsection{Seismic inputs}

A number of $48+48$ seismic records, taken from the ESM database [37], has been considered for the stochastic analyses. The number is doubled because each of the 48 seismic records has been also changed in sign and used as second set of inputs. Among the 48 accelerograms, 24 were registered in Central Italy, whereas 24 were taken from [31]. Several significant intensity measures (IMs) can be associated to each accelerogram. It is important to point out that numerous IMs - acceleration, velocity and energy based - can be calculated for each of them. Nevertheless, in this contribution the focus is on peak ground acceleration (PGA), on Arias intensity $\left(\mathrm{I}_{\mathrm{a}}\right)$ and on peak ground velocity $(\mathrm{PGV})$, the most common IMs referred to in seismic vulnerability analysis. PGV is in particular an IM very significant for rocking blocks. The seismic inputs considered in the analysis are listed in Table 3.

\begin{tabular}{|c|c|c|c|c|c|}
\hline n. & $\begin{array}{l}\text { waveform ID } \\
{\left[\text { yyyymmdd-hhmm-SSS }{ }^{1}-\mathrm{DD}^{2}\right]}\end{array}$ & site & $\begin{array}{c}\mathbf{M}_{\mathbf{L}} \\
{[-]}\end{array}$ & $\begin{array}{r}\text { PGA } \\
{[\mathrm{g}]}\end{array}$ & $\begin{array}{r}\text { PGV } \\
{[\mathrm{cm} / \mathrm{s}]}\end{array}$ \\
\hline 1 & 20160824-0136-AMT-WE & Accumoli & 6.0 & 0.87 & 43.55 \\
\hline 2 & 20160824-0136-NRC-NS & Accumoli & 6.0 & 0.37 & 29.75 \\
\hline 3 & 20160824-0233-FOC-WE & Norcia & 5.4 & 0.18 & 2.79 \\
\hline 4 & 20160824-0233-NRC-NS & Norcia & 5.4 & 0.19 & 9.80 \\
\hline 5 & 20160826-0428-AMT-NS & Amatrice & 4.7 & 0.34 & 11.00 \\
\hline 6 & 20160826-0428-PCB-NS & Amatrice & 4.7 & 0.31 & 5.50 \\
\hline 7 & 20161026-1710-CMI-WE & C.S.A. sul Nera & 5.4 & 0.72 & 55.70 \\
\hline 8 & 20161026-1710-CNE-WE & C.S.A. sul Nera & 5.4 & 0.56 & 17.34 \\
\hline 9 & 20161026-1918-CMI-WE & Visso & 5.9 & 0.65 & 43.76 \\
\hline 10 & 20161026-1918-FOC-WE & Visso & 5.9 & 0.62 & 20.00 \\
\hline 11 & 20161030-0640-MZ24-WE & Norcia & 6.1 & 1.02 & 73.60 \\
\hline 12 & 20161030-0640-MZ51-NS & Norcia & 6.1 & 0.96 & 74.98 \\
\hline 13 & 20161101-0756-MMO-NS & Visso & 4.7 & 0.19 & 7.58 \\
\hline 14 & 20161101-0756-RQT-WE & Visso & 4.7 & 0.13 & 2.80 \\
\hline 15 & 20161103-0035-PBN-NS & P. Torina & 4.8 & 0.31 & 8.47 \\
\hline 16 & 20161103-0035-T1219-WE & P. Torina & 4.8 & 0.34 & 7.88 \\
\hline 17 & 20170118-0925-AMT-NS & Capitignano & 5.4 & 0.35 & 13.15 \\
\hline 18 & 20170118-0925-PCB-NS & Capitignano & 5.4 & 0.18 & 3.79 \\
\hline 19 & 20170118-1014-AMT-NS & Capitignano & 5.4 & 0.32 & 16.10 \\
\hline 20 & 20170118-1014-PCB-NS & Capitignano & 5.4 & 0.60 & 21.02 \\
\hline 21 & 20170118-1025-MSCT-NS & Montereale & 5.3 & 0.28 & 17.03 \\
\hline 22 & 20170118-1025-PCB-NS & Montereale & 5.3 & 0.56 & 19.35 \\
\hline 23 & 20170118-1333-MSCT-NS & C. Amiterno & 5.1 & 0.16 & 6.27 \\
\hline 24 & 20170118-1333-PCB-NS & C. Amiterno & 5.1 & 0.29 & 5.55 \\
\hline $25-48$ & records [51 - 74], Table 5, in Gi & esini et al. 2018 & $>5.5$ & var & $>45$ \\
\hline
\end{tabular}

${ }^{1}$ SSS: station code.

${ }^{2}$ DD: earthquake direction (North-South, NS; West-East, WE).

Table 3: Seismic inputs used in the analysis. 


\subsection{Single realizations of the non-linear dynamic analyses and correlation coefficients}

The single realizations coming from the rocking analyses are performed for both geometries and for the ten cases (free-standing and nine types of tie-rods as described in 3.2). A multistripe analysis [23] is carried out considering six values of PGA (hazard site: Carlentini, Italy [38], [39]). For each PGA value 1920 analyses have been performed, so that 11520 runs resulted in total. An extract of the graphs for the free-standing and tied case of the monumental façade $\left(f_{y}=\right.$ 235, $\varphi=11 \mathrm{~mm}, \varepsilon_{u}=10 \%$ ) is reported in Figure 2. In the vertical axis of the graphs the maximum engineering demand parameter is reported for each rocking analysis with fixed seismic input. Each circle represents the maximum response of single realization obtained from each seismic input. Each accelerogram has a corresponding pair of PGA and PGV displayed in the abscissa axes; each one is scaled for six values of PGA corresponding to specific return periods of the selected construction site. It is evident that when the wall is not restrained by any tie-rod the normalized rotation is greater, and overturning frequently attained (Figure $2 \mathrm{a}$ ). In case the wall is horizontally restrained by a ductile tie-rod of medium strength $\left(\varepsilon_{u}=10 \%, f_{y}=235 \mathrm{MPa}\right.$, $\varphi=11 \mathrm{~mm}$ ), the non-rocking cases are more numerous and the number of overturning cases is only three. Analyzing the results, the correlations coefficients can be easily calculated.

The best correlation is always obtained considering the PGA, with the exception of the monumental unrestrained wall, for which the best IM is the Arias Intensity $\mathrm{I}_{\mathrm{a}}$.

For the same cases displayed in Figure 2, Figure 3 reports the estimated curve with the correlation coefficients:

- $\quad b$-coefficient (Eq. (8)), for the practicality;

- dispersion coefficient $\beta_{D \mid I M}$, for the efficiency;

- $\quad \zeta=\beta_{D \mid I M} / b$, for the proficiency;

- Coefficient of determination $R^{2}$, defined as $1-\frac{\sum\left(\ln \mathrm{D}-\ln \mathrm{S}_{\mathrm{D}}\right)^{2}}{\sum(\ln \mathrm{D}-\operatorname{mean}(\ln \mathrm{D}))^{2}}$;

The correlation is made only for the cases of safe rocking, namely for $1 \mathrm{e}-4<\vartheta / \alpha<1.5$. That is the reason why Figure $3 \mathrm{~b}$ shows only two values of PGA for multistripe analysis (see also for comparison the blue circles in Figure $2 b$ ). Therefore, the probability of exceedance of a specific limit state is calculated only for the safe-rocking cases.

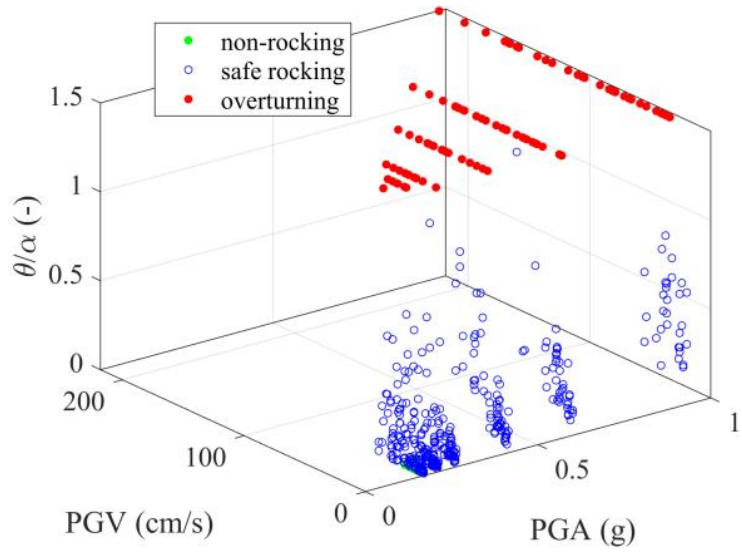

(a)

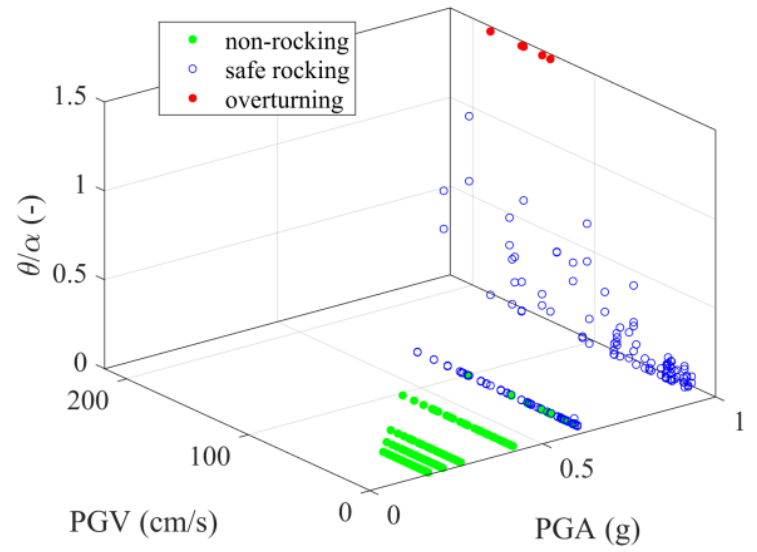

(b)

Figure 2: Realizations of the rocking analysis for specific values of PGA and PGV for unrestrained (a) and tied (b) monumental wall. 
Looking at the results, it is possible to observe that, when the correlation is made towards the PGA (Figure 3a, b), the unrestrained case is worse correlated than the tied case in terms of efficiency and proficiency. As for practicality, the unrestrained case is better correlated. The results in terms of Arias Intensity, for which the correlation is the most efficient for the unrestrained case, are scattered because this intensity measure is calculated after that the PGA of each accelerogram is scaled according to the formula given in [31]. the unrestrained case is worse correlated than the tied case in terms of efficiency and proficiency (Figure 3c, d). Concerning practicality - and also proficiency - the unrestrained case is again better correlated than the restrained case. Since the dispersion coefficient for the unrestrained case is slightly greater than that for the tied case, the correlation of the first one is more efficient. The maximum coefficient of determination is observed for the correlation of PGA (restrained case) and of $I_{a}$ (unrestrained case) and is equal to 0.57 for both scenarios (Figure $3 b, c$ ).

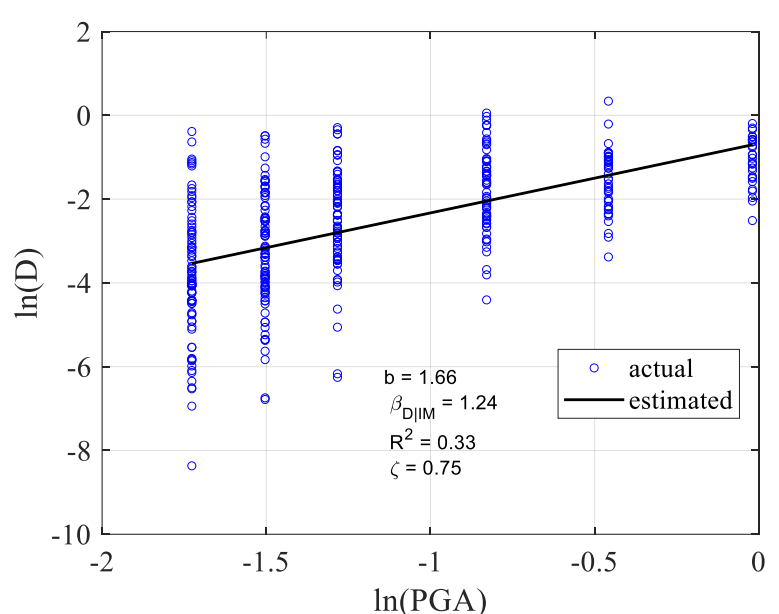

(a)

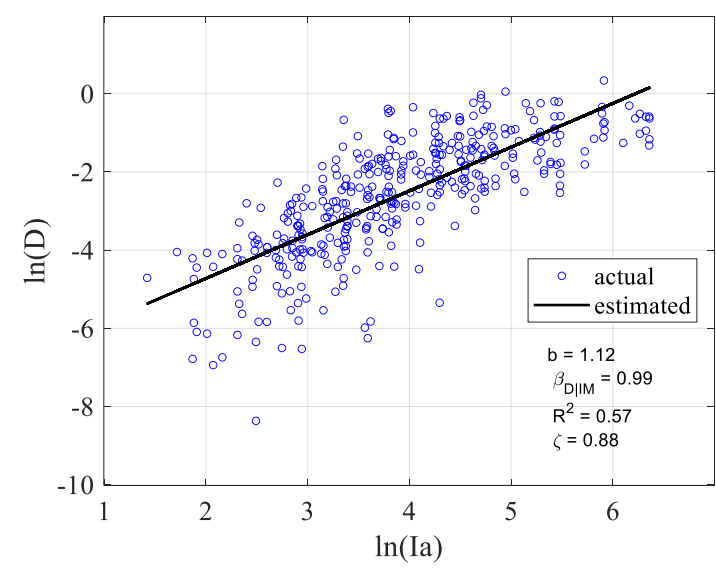

(c)

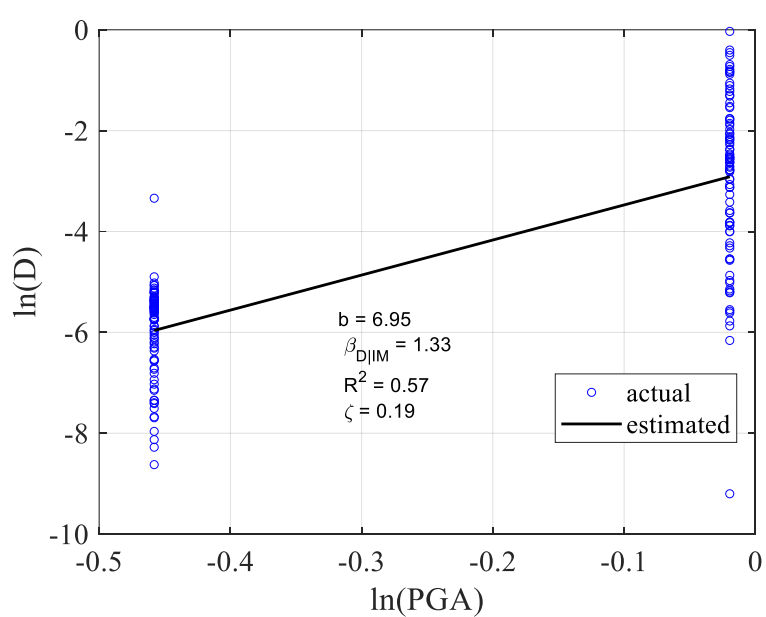

(b)

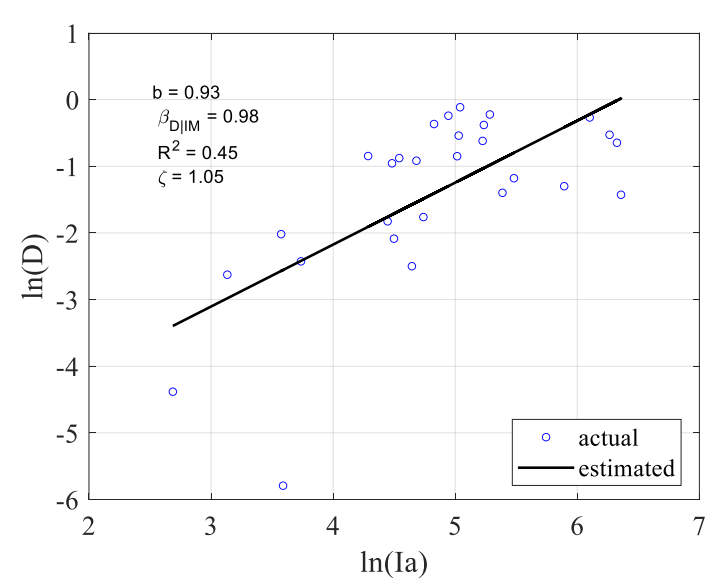

(d)

Figure 3: Structural demand and correlation coefficients for unrestrained $(\mathrm{a}, \mathrm{c})$ and tied $(\mathrm{b}, \mathrm{d})$ monumental wall.

\section{FRAGILITY CURVES}

This section discusses the fragility curves obtained for all the cases with the calculation process described in section 2.2. A first comparison can be made between the ordinary and the monumental wall. Their slenderness value is the same (height to thickness $=10$ ) but the size of 
the ordinary wall is half of that monumental. The fragility curves representing the conditional probability of the EDP conditioned to PGA are reported in Figure 4 for all the five limit states. The probability of occurrence of the rocking initiation state is much less for the ordinary wall rather than that of the monumental wall. For the ordinary wall, of small size, there is a narrower difference between probability of occurrence of the limit states, from the rocking initiation to the near-collapse. The ordinary wall appears more stable than the monumental wall, although the slenderness values are the same. In this case, therefore, the common scale effect usually exhibited by free rocking blocks is not observed.

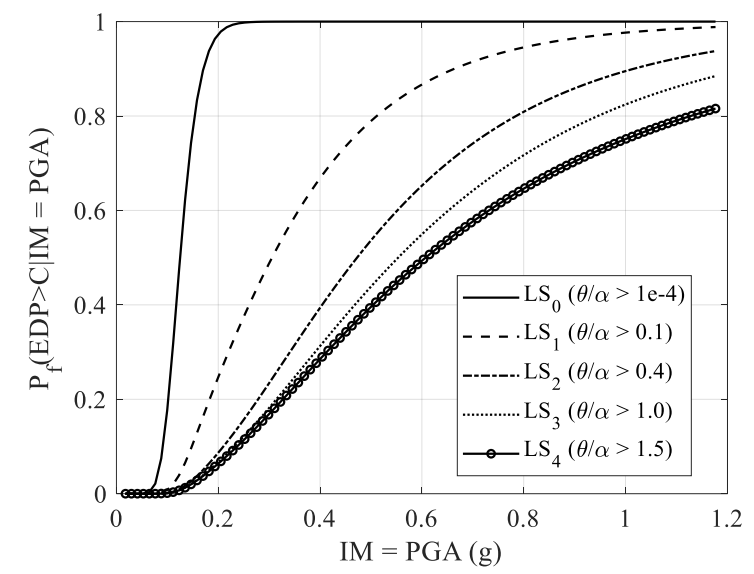

(a)

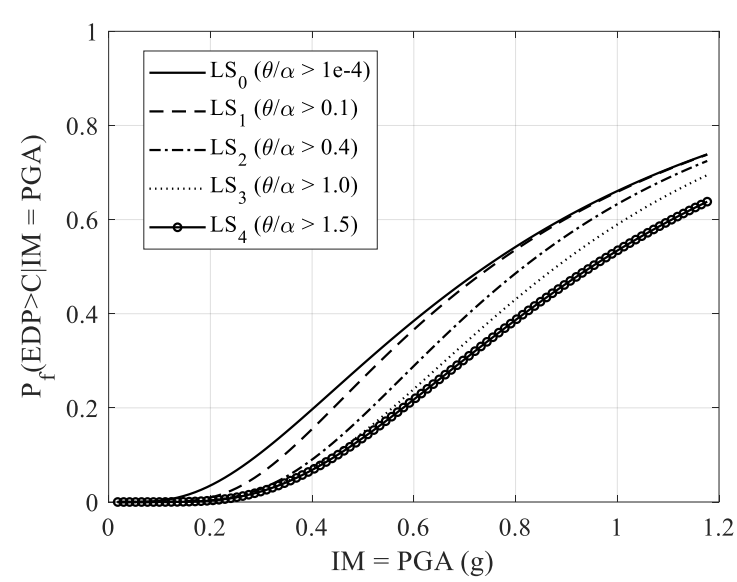

(b)

Figure 4: Fragility curves for the unrestrained monumental (a) and ordinary (b) wall.

Considering the monumental wall, the fragility curves considering again the five limit states but with different values of ductility capacity of the tie-rod are displayed in Figure 5. The subfigures respectively indicate the response for the unrestrained wall (a), the wall restrained by a brittle tie-rod with $\varepsilon_{u}=0 \%$ (b), by a low ductility tie-rod with $\varepsilon_{u}=2 \%$ (c), by a high ductility tierod with $\varepsilon_{u}=10 \%(\mathrm{~d})$. It is evident that a more ductile tie-rod reduces the probability of overcoming the attainment of an EDP threshold. The case with the most ductile tie-rod results in flatter fragility curves which lose their typical s-shape, which indicate greater uncertainty.

For the medium steel axial strength (235 $\mathrm{MPa}, \varphi=11 \mathrm{~mm})$, the probability of exceedance for the limited rocking state $\left(\mathrm{LS}_{1}\right)$ is reported in Figure 6a: the most dangerous case is the brittle tie-rod, as expected. The probability of exceedance of the limited rocking state is respectively by $85 \%, 30 \%, 8 \%$ and $3 \%$ for unrestrained wall, wall restrained by brittle, ductile and lowductile tie rod for PGA=0.8 g. PGA values lower than it, no remarkable difference is observed in terms of probability of exceedance, since the tie-rod impedes a considerable wall rocking. The trend of reduction of probability of exceedance above mentioned, not fully expected for ductile and low-ductile tie-rod, depends on the higher uncertainty that affects the probability of exceedance of a ductile tie-rod. So, even though the curve related to the ductile tie-rod is not considered, the reduction of probability of exceedance is sensitively strong being an order of magnitude lower for the low-ductile tie-rod (Figure 6a). Therefore, it is extremely beneficial to restrain the wall even with a low-ductile (but also a brittle) tie-rod. The choice will depend on the assumed probability of exceedance depending on the return periods established by the standards. 


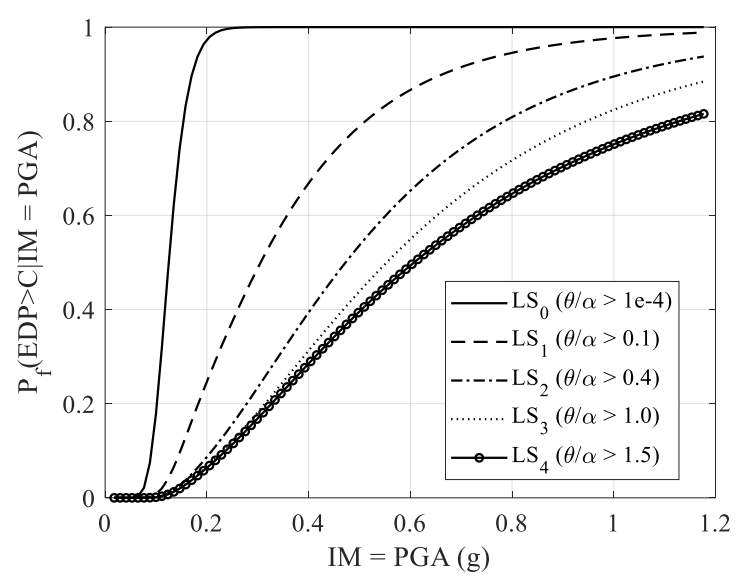

(a)

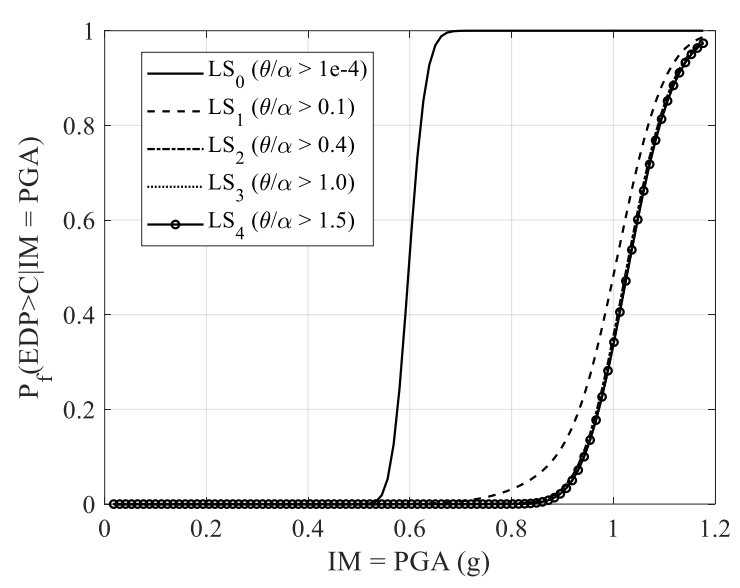

(c)

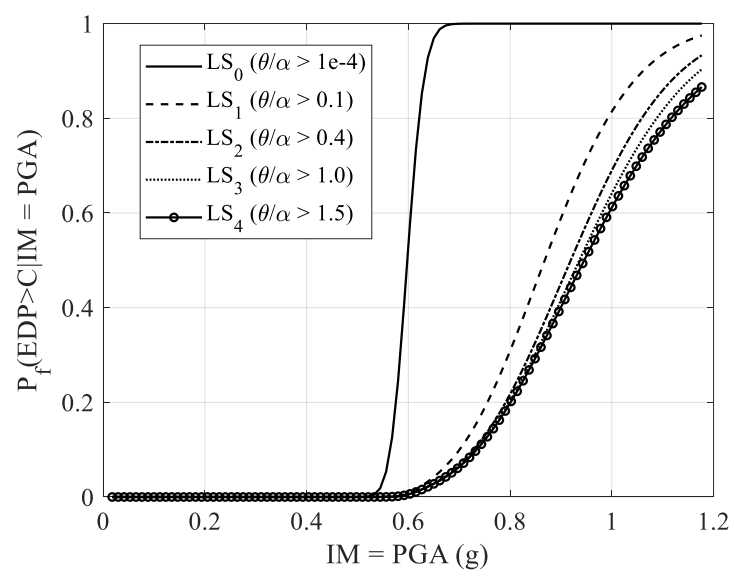

(b)

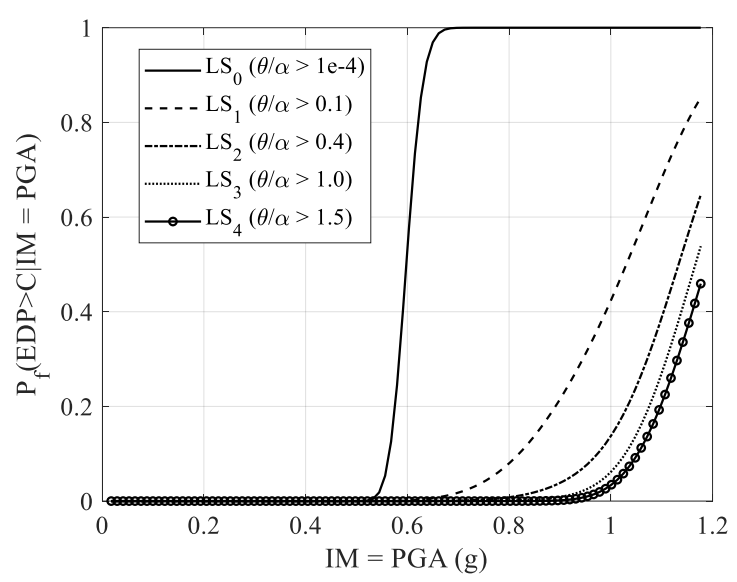

(d)

Figure 5: Fragility curves for the monumental wall: unrestrained (a); brittle (b); low ductility (c) and ductile (d) tie-rod.

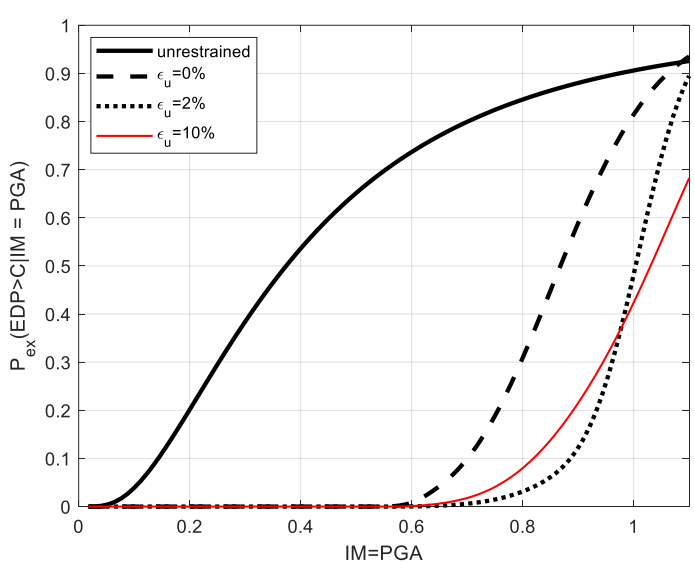

(a)

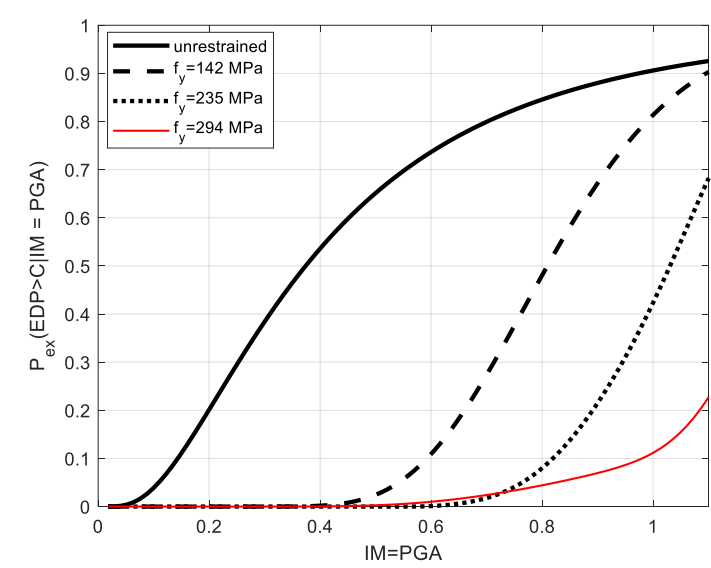

(b)

Figure 6: Fragility curves for monumental wall: parametric analyses in terms of (a) steel ductility and (b) axial strength for the limited rocking state (LS1). 
As for the variation of steel strength, keeping constant the tie-rod ultimate strain to $10 \%$, the weaker material has an increased probability of exceedance that anyway remains low (about $10 \%$ for $\mathrm{PGA}=0.6 \mathrm{~g}$ ) even for medium-high intensity earthquakes. As a consequence, in many cases even a weak tie steel can be really beneficial for the reduction of the probability of exceedance of the limited rocking state. The cases of medium-high strength ties (235 MPa, $\varphi=$ $11 \mathrm{~mm}$ and $294 \mathrm{MPa}, \varphi=10 \mathrm{~mm}$ ) give coincident fragility curves since the stress of $235 \mathrm{MPa}$ is never overcome.

\section{SEISMIC DEMAND HAZARD CURVES}

The seismic demand hazard curves are obtained by following the procedure described in [23]. The output is readable as annual exceedance rate $\lambda_{E D P}(e d p)$ for the five limit states indicated in the abscissa axis $(\vartheta / \alpha=1 \mathrm{E}-4,0.1,0.4,1.0,1.5)$. This information is more complete than the mere fragility curves, as the demand hazard is considered in the probabilistic assessment. The seismic demand hazard curve is much more conservative in case of restrained condition than for the unrestrained monumental wall (Figure 7a). Considering the same steel strength ( $235 \mathrm{MPa},, \varphi=11 \mathrm{~mm}$ ) and varying the ultimate strain of the tie-rod (Figure $7 \mathrm{~b}$ ), it is clear that the annual exceedance rate is the lowest in case of low ductile tie-rod behavior than in case of brittle behavior. Moreover, a brittle behavior of the tie-rod could lead to unexpected performance of the tie-rod especially in case of historical ties, where localized failures can occur [36]. Thus, it is recommended to prefer even a low-ductile behavior that strongly reduces the seismic demand hazard curves. Indeed, for instance considering the moderate rocking limit state $(\vartheta / \alpha=0.4)$, the annual exceedance rate decreases by over $60 \%$ passing from a brittle to a lowductile tie rod. Again the red curve, related to a highly ductile tie-rod, for same ranges of EDP exhibits unexpected performance. In any case, such a behavior can be neglected being the corresponding fragility curves characterized by greater uncertainties.

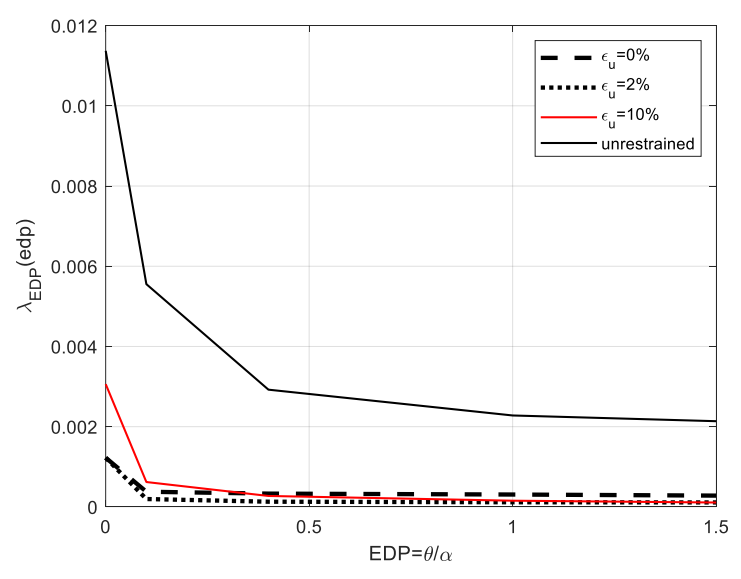

(a)

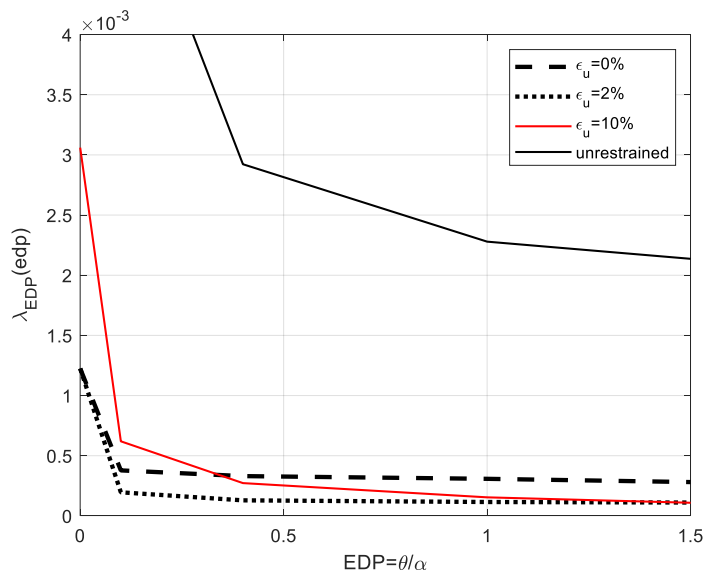

(b)

Figure 7: Seismic hazard curves for the monumental wall: parametric analyses in terms of steel ductility (a) with a detail for the restrained case (b).

Discussing how the tie-rod strength influences the seismic risk, it is evident that, as observed for the fragility curves, there is not a sensitive change in the annual rate of exceedance whether a high-strength tie-rod is assumed or a medium strength is considered (Figure 8).

Referring to the moderate rocking limit state $(\vartheta / \alpha=0.4)$, the annual exceedance rates are:

- $1.8 \mathrm{e}-5$ for the strength values of $294 \mathrm{MPa}, \varphi=10 \mathrm{~mm}$; 
- 7.7 e-5 for $235 \mathrm{MPa}$;

- 2.7e-4 for $142 \mathrm{MPa}$;

- $\quad 2.9 \mathrm{e}-3$ for the unrestrained case.

They respectively correspond to the fact that this limit state is exceeded once every 56000 years (high tie-rod strength) 13000 (medium), 3700 (low) and 345 years. Therefore, even with a low-strength tie-rod, the rate of occurrence is reduced.

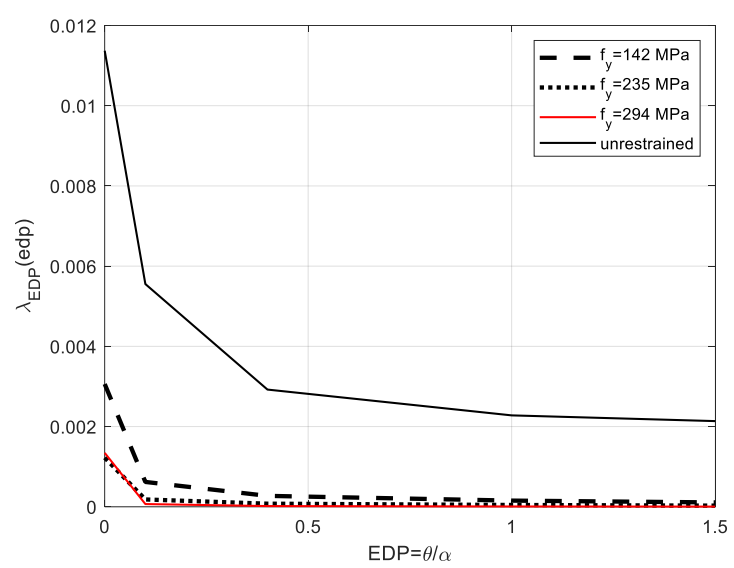

(a)

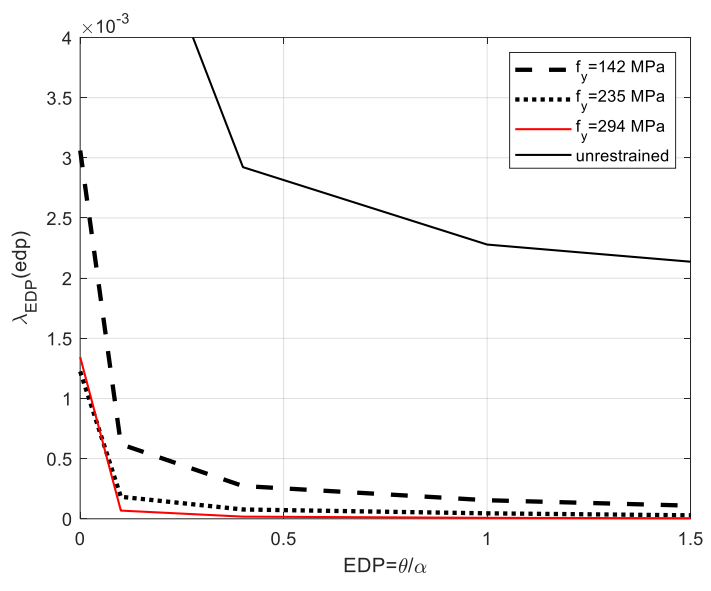

(b)

Figure 8: Seismic hazard curves for the monumental wall: parametric analyses in terms of steel strength (a) with a detail for the restrained case (b).

Taking into account the severe rocking $(\vartheta / \alpha=1.0)$, the annual exceedance rates are:

- $8 \mathrm{e}-6$ for the strength value of $294 \mathrm{MPa}, \varphi=10 \mathrm{~mm}$ (one occurrence every 125000 years);

- $\quad 4.5 \mathrm{e}-5$ for $235 \mathrm{MPa}(22000$ years);

- $\quad 1.5 \mathrm{e}-4$ for $142 \mathrm{MPa}(2000$ years);

- $\quad 2.2 \mathrm{e}-3$ for the unrestrained case (450 years).

Obviously, the two latter cases are over-conservative considering the life cycle of a masonry building, therefore even small strength values are sufficient to guarantee a remarkable increase of safety. For the severe rocking limit state, the reduction of annual exceedance rate is less evident than that for the moderate rocking limit state.

\section{CONCLUSIONS}

This paper discussed the role of tie-rods in the dynamic response of restrained masonry walls subjected to earthquakes. The analysis was performed by following a fully probabilistic approach with a multi-stripe analysis obtaining seismic fragility and hazard curves. Two typical masonry wall, one ordinary and the other of monumental type, were taken into account as unrestrained and restrained by tie-rods of different mechanical properties. Considering the results of over 11500 analyses, the best correlated intensity measures were observed to be peak ground acceleration and Arias Intensity.

Observing the fragility curves, the most dangerous case is the brittle tie-rod, as expected. The probability of exceedance of the limited rocking state is respectively by $85 \%, 30 \%, 8 \%$ and $3 \%$ for unrestrained wall, wall restrained by brittle, ductile and low-ductile tie rod for PGA $=0.8$ 
$\mathrm{g}$. The reduction of probability of exceedance is significant being an order of magnitude lower for the case of low-ductile tie-rod with respect to the case of brittle tie-rod. Therefore, it is extremely beneficial to restrain the wall even with a low-ductile (but also a brittle, in comparison with an unrestrained wall) tie-rod. The choice of a specific tie-rod will depend on the admissible probability of exceedance depending on the return periods established by the standards.

As for the variation of steel strength, keeping constant the tie rod ultimate strain to $10 \%$, the weaker material has an increased probability of exceedance that anyway keeps low (about 10\% for $\mathrm{PGA}=0.6 \mathrm{~g}$ ) even for medium-high intensity earthquakes. As a consequence, in many cases even a weak steel can be really beneficial for the reduction of the probability of exceedance of the limited rocking state.

For what concerns the seismic hazard demand curves, it is preferable for the tie-rod a lowductile rather than a brittle behavior that strongly reduces the seismic demand hazard curves. A highly ductile performance could cause more uncertainties in the results. Considering the moderate rocking limit state, the annual exceedance rate decreases by over $60 \%$ passing from a brittle to a low-ductile tie rod. As for the influence of the tie-rod strength, even a low-medium values produce a remarkable reduction of annual exceedance rate. For instance, a sever rocking limit state occurs for the unrestrained monumental wall every 450 years and every 2000 years for the wall restrained by a tie rod of strength $142 \mathrm{MPa}$ and a diameter of $14 \mathrm{~mm}$. The return period of the same event increases to 22000 years for a strength of $235 \mathrm{MPa}$ (and diameter 11 $\mathrm{mm}$ ) and to 125000 years for $292 \mathrm{MPa}$ (diameter $10 \mathrm{~mm}$ ). Obviously, the two latter cases are over-conservative considering the life cycle of a masonry building, therefore even small strength values are sufficient to guarantee a remarkable increase of safety.

Finally, the probabilistic approach described here for the seismic assessment of the out-ofplane of masonry walls is able to reliably predict the seismic demand hazard curves to define the most appropriate type of steel tie rod to use as anti-seismic device.

\section{REFERENCES}

[1] M. Sassu, L. Giresini, E. Bonannini, and M. L. Puppio, On the Use of Vibro-Compressed Units with Bio-Natural Aggregate. Buildings, 6, 2016.

[2] M. L. Puppio and L. Giresini, Estimation of tensile mechanical parameters of existing masonry through the analysis of the collapse of Volterra's urban walls. Frattura ed Integrità Strutturale, 13, 2019.

[3] F. Solarino, D. Oliveira, and L. Giresini, Wall-to-horizontal diaphragm connections in historical buildings: A state-of-the-art review. Engineering Structures, 199, 2019.

[4] L. Giresini and M. Sassu, Horizontally restrained rocking blocks: evaluation of the role of boundary conditions with static and dynamic approaches. Bulletin of Earthquake Engineering, 15, 385-410, 2017.

[5] L. Giresini, Design strategy for the rocking stability of horizontally restrained masonry walls. , in COMPDYN 2017 6th ECCOMAS Thematic Conference on Computational Methods in Structural Dynamics and Earthquake Engineering, Rhodes Island, Greece, 15-17 June 2017., 2017.

[6] N. Makris and M. F. Vassiliou, Dynamics of the Rocking Frame with Vertical Restrainers. Journal of Structural Engineering, 141, 2015.

[7] C. Casapulla, P. Jossa, and A. Maione, Rocking motion of a masonry rigid block under seismic actions: A new strategy based on the progressive correction of the resonance 
response | Il moto sotto sisma del blocco murario: Analisi per progressiva correzione della risposta in risonanza. Ingegneria Sismica, 27, 35-48, 2010.

[8] C. Casapulla and A. Maione, Critical Response of Free-Standing Rocking Blocks to the Intense Phase of an Earthquake. International Review of Civil Engineering, 8, 1-10, 2017.

[9] C. Casapulla and A. Maione, Free Damped Vibrations of Rocking Rigid Blocks as Uniformly Accelerated Motions. International Journal of Structural Stability and Dynamics, 17, 1-19, 2016.

[10] L. Giresini, M. Sassu, and L. Sorrentino, In situ free-vibration tests on unrestrained and restrained rocking masonry walls. Earthquake Engineering \& Structural Dynamics, 47, 3006-3025, 2018.

[11] L. Giresini, M. L. Puppio, and F. Taddei, Experimental pull-out tests and design indications for strength anchors installed in masonry walls. Materials and Structures, 2020.

[12] H. Derakhshan, M. C. Griffith, and J. M. Ingham, Out-of-plane seismic response of vertically spanning URM walls connected to flexible diaphragms. Earthquake Engineering \& Structural Dynamics, 45, 563-580, 2016.

[13] C. Casapulla and F. Portioli, Experimental tests on the limit states of dry-jointed tuff blocks. Materials and Structures/Materiaux et Constructions, 49, 751-767, 2016.

[14] L. Giresini, F. Solarino, O. Paganelli, D. V. Oliveira, and M. Froli, One-sided rocking analysis of corner mechanisms in masonry structures: influence of geometry, energy dissipation, boundary conditions. Soil Dynamics and Earthquake Engineering, 123, 357370, 2019.

[15] C. Casapulla, L. Giresini, L. U. Argiento, and A. Maione, Nonlinear Static and Dynamic Analysis of Rocking Masonry Corners Using Rigid Macro-Block Modeling. International Journal of Structural Stability and Dynamics, 19, 1950137, 2019.

[16] L. U. Argiento, A. Maione, and L. Giresini, The corner failure in a masonry building damaged by the 2016-2017 central Italy earthquake sequence., in COMPDYN 2019 7th ECCOMAS Thematic Conference on Computational Methods in Structural Dynamics and Earthquake Engineering, 2019, 633-650, .

[17] L. Giresini, B. Pantò, S. Caddemi, and I. Caliò, Out-of-plane seismic response of masonry façades using discrete macro-element and rigid block models. , in COMPDYN 2019 7th ECCOMAS Thematic Conference on Computational Methods in Structural Dynamics and Earthquake Engineering, 2019, 702-717, .

[18] C. Casapulla, A. Maione, L. U. Argiento, and E. Speranza, Corner failure in masonry buildings: An updated macro-modeling approach with frictional resistances. European Journal of Mechanics, A/Solids, 70, 2018.

[19] A. De Falco, L. Giresini, and M. Sassu, Temporary preventive seismic reinforcements on historic churches: numerical modeling of San Frediano in Pisa. Applied Mechanics and Materials, 351, 1393-1396, 2013.

[20] L. Giresini and C. Butenweg, Earthquake Resistant Design of Structures According to Eurocode 8., in Structural Dynamics with Applications in Earthquake and Wind Engineering, 2019, 197-358, . 
[21] M. Andreini, A. De Falco, L. Giresini, and M. Sassu, Collapse of the historic city walls of Pistoia (Italy): causes and possible interventions. Applied Mechanics and Materials, 351-352, 2013.

[22] O. AlShawa, D. Liberatore, and L. Sorrentino, Dynamic One-Sided Out-Of-Plane Behavior of Unreinforced-Masonry Wall Restrained by Elasto-Plastic Tie-Rods. International Journal of Architectural Heritage , 13, 340-357, 2019.

[23] B. A. Bradley, The seismic demand hazard and importance of the conditioning intensity measure. Earthquake Engineering \& Structural Dynamics, 41, 1417-1437, 2012.

[24] P. Croce, F. Landi, and P. Formichi, Probabilistic Seismic Assessment of Existing Masonry Buildings. Buildings, 9, 2019.

[25] P. Croce, M. L. Beconcini, P. Formichi, F. Landi, B. Puccini, and V. Zotti, Bayesian Methodology for Probabilistic Description of Mechanical Parameters of Masonry Walls. ASCE-ASME Journal of Risk and Uncertainty in Engineering Systems, Part A: Civil Engineering, 7, 4021008, 2021.

[26] M. L. Beconcini, P. Croce, F. Marsili, M. Muzzi, and E. Rosso, Probabilistic reliability assessment of a heritage structure under horizontal loads. Probabilistic Engineering Mechanics, 45, 198-211, 2016.

[27] P. Croce, M. L. Beconcini, P. Formichi, F. Landi, B. Puccini, and V. Zotti, Seismic risk evaluation of existing masonry buildings: Methods and uncertainties. , in 20th Congress of IABSE, New York City 2019: The Evolving Metropolis - Report 2019, 2019, 25102515, .

[28] L. Giresini, F. Solarino, F. Taddei, G. Mueller, and P. Croce, Influence of stiffness and damping parameters of seismic passive control devices in one-sided rocking of masonry walls. submitted to Journal of Structural Engineering, 2021.

[29] Giresini L., Sassu M., Horizontally restrained rocking blocks: evaluation of the role of boundary conditions with static and dynamic approaches. Bulletin of Earthquake Engineering, 15, 385-410, 2017.

[30] MATLAB. version 9.4.0.813654 (R2018a). The MathWorks Inc., Natick, Massachusetts, 2018. . .

[31] L. Giresini, C. Casapulla, R. Denysiuk, J. Matos, and M. Sassu, Fragility curves for free and restrained rocking masonry façades in one-sided motion. Engineering Structures, 164, 195-213, 2018.

[32] J. E. Padgett, B. G. Nielson, and R. DesRoches, Selection of optimal intensity measures in probabilistic seismic demand models of highway bridge portfolios. Earthquake Engineering \& Structural Dynamics, 37, 711-725, 2008.

[33] L. Giresini, F. Taddei, C. Casapulla, and G. Mueller, Stochastic assessment of rocking masonry façades under real seismic records., in COMPDYN 2019 7th ECCOMAS Thematic Conference on Computational Methods in Structural Dynamics and Earthquake Engineering, 2019, 673-689, .

[34] F. Solarino and L. Giresini, Fragility curves and seismic demand hazard analysis of rocking walls restrained elasto-plastic ties. Earthquake Eng. Struct. Dyn. (submitted to), 2021.

[35] Ministero delle infrastrutture e dei trasporti, Circolare applicativa 21 gennaio 2019, $n$. 
7, Istruzioni per l'applicazione dell'"Aggiornamento delle "Norme tecniche per le costruzioni”"». In Italian. 2019.

[36] C. Calderini, R. Vecchiattini, C. Battini, and P. Piccardo, Mechanical and metallographic characterization of iron tie-rods in masonry buildings: An experimental study., in Proceedings of the 10th International Conference on Structural Analysis of Historical Constructions, SAHC, Leuven, Belgium, September 13-15 2016, 2016, 1293-300, .

[37] L. Luzi, R. Puglia, E. Russo, and O. WG5, Engineering Strong Motion Database, version 1.0, Istituto Nazionale di Geofisica e Vulcanologia, Observatories \& Research Facilities for European Seismology. . 2016.

[38] General Assembly of the Superior Council of Public, Circolare attuativa del D.M. 17/01/2018 (NTC 2018). 2019.

[39] Decreto Ministeriale 17/01/2018, Italian Technical Standards for buildings (Nuove Norme Tecniche per le Costruzioni, in italian). . 2018. 